Communications in Physics, Vol. 23, No. 1 (2013), pp. 1-9

\title{
PHENOMENOLOGY OF THE REDUCED MINIMAL 3-3-1 MODEL
}

\author{
TRAN THANH LAM, NGUYEN CHI THAO, HO HOANG TINH, \\ NGUYEN THI XINH, AND NGUYEN VAN XUYEN \\ Department of Physics, Can Tho University, Can Tho, Vietnam
}

HOANG NGOC LONG

Institute of Physics, VAST

\begin{abstract}
The detailed analysis of the gauge model based on $\mathrm{SU}(3)_{C} \otimes \mathrm{SU}(3)_{L} \otimes \mathrm{U}(1)_{X}$ group with minimal content of lepton and Higgs is presented. It is shown that with just two Higgs triplets, all fermions and gauge bosons can get correct masses. The advantage of the model under consideration is that a huge number of free parameters is reduced, and the model's predictiveness is much improved.
\end{abstract}

\section{INTRODUCTION}

The experimental evidences of nonzero neutrino masses and mixing [1] have shown that the standard model (SM) of fundamental particles and interactions must be extended. Among many extensions of the SM known today, the models based on gauge symmetry $\mathrm{SU}(3)_{C} \otimes \mathrm{SU}(3)_{L} \otimes \mathrm{U}(1)_{X}$ (called 3-3-1 models) [2,3] has interesting features. First, $\left[\mathrm{SU}(3)_{L}\right]^{3}$ anomaly cancelation requires that the number of $\mathrm{SU}(3)_{L}$ fermion triplets must equal to that of antitriplets. If these multiplets are respectively enlarged from those of the SM, the fermion family number is deduced to be a multiple of the fundamental color number, which is three, coinciding with the observation. In addition, one family of quarks has to transform under $\mathrm{SU}(3)_{L}$ differently from the other two. This can lead to an explanation why the top quark is uncharacteristically heavy.

One of the weaknesses of the mentioned 3-3-1 models that reduces their predictive possibility is a plenty or complication in the scalar sectors. The attempt on this direction to realize simpler scalar sectors has recently been constructed 3-3-1 model with minimal Higgs sector called the economical 3-3-1 model [4,5]. The 3-3-1 model with minimal content of fermions and Higgs sector (called the reduced minimal (RM) 3-3-1 model) has also been constructed in [6].

\section{PARTICLE CONTENT}

The fermion content of the model under consideration is the same as in the minimal 3-3-1 model [2]. The left-handed leptons and quarks transform under the $\mathrm{SU}(3)_{L}$ gauge 
group as the triplets

$$
\begin{aligned}
f_{a L} & =\left(\begin{array}{c}
\nu_{\ell_{a}} \\
\ell_{a} \\
\ell_{a}^{c}
\end{array}\right)_{L} \sim(3,0) \quad Q_{1 L}=\left(\begin{array}{c}
u_{1} \\
d_{1} \\
J_{1}
\end{array}\right)_{L} \sim\left(3, \frac{2}{3}\right), \\
Q_{i L} & =\left(\begin{array}{c}
d_{i} \\
-u_{i} \\
J_{i}
\end{array}\right)_{L} \sim\left(\mathbf{3}^{*},-\frac{1}{3}\right)
\end{aligned}
$$

where $a=1,2,3, l_{1}=e, l_{2}=\mu, l_{3}=\tau$ and $i=2,3$. The $J_{1}$ exotic quark carries $5 / 3$ units of elementary electric charge, while $J_{2}$ and $J_{3}$ carry $-4 / 3$ each one. In Eqs. (1) the numbers $0,2 / 3$, and $-1 / 3$ are the $\mathrm{U}(1)_{X}$ charges. The right-handed quarks are singlets of the $\mathrm{SU}(3)_{L}$ group,

$$
\begin{aligned}
& u_{R}^{a} \sim(\mathbf{1}, 2 / 3), \quad d_{R}^{a} \sim(\mathbf{1},-1 / 3), a=1,2,3, \\
& J_{1 R} \sim(\mathbf{1}, 5 / 3), \quad J_{i R} \sim(\mathbf{1},-4 / 3) .
\end{aligned}
$$

The charge operator is defined by

$$
\frac{Q}{e}=\frac{\lambda_{3}}{2}-\frac{\sqrt{3}}{2} \lambda_{8}+X
$$

where $\lambda_{3}$ and $\lambda_{8}$ are the diagonal Gell-Mann matrices. Note that for antitriplet, we have to replace the Gell-mann matrix by $\bar{\lambda}=-\lambda^{*}$.

The two Higgs scalar triplets

$$
\rho=\left(\begin{array}{c}
\rho^{+} \\
\rho^{0} \\
\rho^{++}
\end{array}\right) \sim(\mathbf{3}, 1), \quad \chi=\left(\begin{array}{c}
\chi^{-} \\
\chi^{--} \\
\chi^{0}
\end{array}\right) \sim(\mathbf{3},-1),
$$

are the minimal content of Higgs sector enough to break the symmetry spontaneously and generate the masses of fermions and gauge bosons in the model. The neutral scalar fields develop the vacuum expectation values (VEVs) $\left\langle\rho^{0}\right\rangle=\frac{v_{\rho}}{\sqrt{2}}$ and $\left\langle\chi^{0}\right\rangle=\frac{v_{\chi}}{\sqrt{2}}$, with $v_{\rho}=246$ $\mathrm{GeV}$.

The pattern of symmetry breaking is

$$
\mathrm{SU}(3)_{L} \otimes \mathrm{U}(1)_{X} \stackrel{\left\langle\chi^{0}\right\rangle}{\longrightarrow} \mathrm{SU}(2)_{L} \otimes \mathrm{U}(1)_{Y} \stackrel{\left\langle\eta^{0}, \rho^{0}\right\rangle}{\longrightarrow} \mathrm{U}(1)_{\mathrm{em}}
$$

and so, we can expect

$$
v_{\chi} \gg v_{\eta}, v_{\rho}
$$

Since lepton and antilepton were put in the same triplet, therefore lepton number is not conserved in this model. It is better to work with a new conserved charge $\mathcal{L}$ commuting with the gauge symmetry $[7,8]$ and related to the ordinary lepton number by diagonal matrices $L=\frac{4}{\sqrt{3}} T_{8}+\mathcal{L}$.

Another useful conserved charge $\mathcal{B}$ is usual baryon number [8] $B=\mathcal{B} I$. These numbers are given $[7,8]$ in the Table 1

In Table 2, we list particles with non-zero lepton number

Table 2 shows that the exotic quarks carry lepton number two. Hence they are bilepton quarks. 
Table 1. $\mathcal{B}$ and $\mathcal{L}$ charges for multiplets in the RM 3-3-1 model.

\begin{tabular}{|c|ccccccccc|}
\hline Multiplet & $\chi$ & $\rho$ & $Q_{1 L}$ & $Q_{i L}$ & $u_{a R}$ & $d_{a R}$ & $J_{1 R}$ & $J_{i R}$ & $f_{a L}$ \\
\hline $\mathcal{B}$ charge & 0 & 0 & $\frac{1}{3}$ & $\frac{1}{3}$ & $\frac{1}{3}$ & $\frac{1}{3}$ & $\frac{1}{3}$ & $\frac{1}{3}$ & 0 \\
\hline $\mathcal{L}$ charge & $\frac{4}{3}$ & $-\frac{2}{3}$ & $-\frac{2}{3}$ & $\frac{2}{3}$ & 0 & 0 & -2 & 2 & $\frac{1}{3}$ \\
\hline
\end{tabular}

Table 2. Nonzero lepton number $L$ of fields in the RM 3-3-1 model .

\begin{tabular}{c|cc|ccc|cccc|}
\hline Fields & $l_{L}^{c}$ & $l_{L}$ & $\rho_{3}^{++}$ & $\chi_{1}^{-}$ & $\chi_{2}^{--}$ & $J_{i L}$ & $J_{i R}$ & $J_{1 L}$ & $J_{1 R}$ \\
\hline$L$ & -1 & 1 & -2 & 2 & 2 & 2 & 2 & -2 & -2 \\
\hline
\end{tabular}

\section{HIGGS POTENTIAL}

The most general renormalizable, gauge and Lorentz invariant scalar potential is

$$
\begin{aligned}
V(\chi, \rho)= & \mu_{1}^{2} \rho^{\dagger} \rho+\mu_{2}^{2} \chi^{\dagger} \chi+\lambda_{1}\left(\rho^{\dagger} \rho\right)^{2}+\lambda_{2}\left(\chi^{\dagger} \chi\right)^{2} \\
& +\lambda_{3}\left(\rho^{\dagger} \rho\right)\left(\chi^{\dagger} \chi\right)+\lambda_{4}\left(\rho^{\dagger} \chi\right)\left(\chi^{\dagger} \rho\right),
\end{aligned}
$$

whose manifest simplicity turns the RM331 model a real gain as compared to the original minimal 3-3-1 model since the number of free parameters is reduced from, at least, thirteen to only six.

Expansion of $\rho^{0}$ and $\chi^{0}$ is

$$
\rho^{0}, \chi^{0} \rightarrow \frac{1}{\sqrt{2}}\left(v_{\rho, \chi}+R_{\rho, \chi}+i I_{\rho, \chi}\right) .
$$

On substituting this expansion in the above potential we obtain the following set of minimum constraint equations,

$$
\begin{aligned}
& \mu_{1}^{2}+\lambda_{1} v_{\rho}^{2}+\frac{\lambda_{3} v_{\chi}^{2}}{2}=0 \\
& \mu_{2}^{2}+\lambda_{2} v_{\chi}^{2}+\frac{\lambda_{3} v_{\rho}^{2}}{2}=0 .
\end{aligned}
$$

This potential immediately gives us two charged Goldstones bosons $\rho^{ \pm}$and $\chi^{ \pm}$which are eaten by the gauge bosons $W^{ \pm}$and $V^{ \pm}$.

In the doubly charged scalars, the mass matrix in the basis $\left(\chi^{++}, \rho^{++}\right)$is given by,

$$
\left(\begin{array}{cc}
\frac{1}{\lambda} v_{4} v_{\chi}^{2} & \frac{1}{2} \lambda_{4} v_{\chi} v_{\rho} \\
\frac{1}{2} \lambda_{4} v_{\chi} v_{\rho} & \frac{1}{2} \lambda_{4} v_{\rho}^{2}
\end{array}\right)
$$

This matrix has the following squared mass eigenvalues,

$$
m_{\tilde{h}^{--}}^{2}=0 \quad \text { and } \quad m_{h^{--}}^{2}=\frac{\lambda_{4}}{2}\left(v_{\chi}^{2}+v_{\rho}^{2}\right)
$$

where the corresponding eigenstates are,

$$
\left(\begin{array}{c}
\tilde{h}^{++} \\
h^{++}
\end{array}\right)=\left(\begin{array}{cc}
c_{\alpha} & -s_{\alpha} \\
s_{\alpha} & c_{\alpha}
\end{array}\right)\left(\begin{array}{c}
\chi^{++} \\
\rho^{++}
\end{array}\right)
$$


with

$$
c_{\alpha}=\frac{v_{\chi}}{\sqrt{v_{\chi}^{2}+v_{\rho}^{2}}}, s_{\alpha}=\frac{v_{\rho}}{\sqrt{v_{\chi}^{2}+v_{\rho}^{2}}} .
$$

It follows that $\tilde{h}^{ \pm \pm}$are the Goldstones eaten by the gauge bosons $U^{ \pm \pm}$, while $h^{ \pm \pm}$ remains as a physical scalar in the spectrum.

Regarding the neutral scalars, their mass matrix takes the following form in the basis $\left(R_{\chi}, R_{\rho}\right)$,

$$
\left(\begin{array}{cc}
\lambda_{2} v_{\chi}^{2} & \frac{1}{2} \lambda_{3} v_{\chi} v_{\rho} \\
\frac{1}{2} \lambda_{3} v_{\chi} v_{\rho} & \lambda_{1} v_{\rho}^{2}
\end{array}\right)
$$

This matrix gives us two eigenvalues

$$
\begin{aligned}
& m_{h_{1}}^{2}=\frac{1}{2} v_{\chi}^{2}\left(\lambda_{1} t^{2}+\lambda_{2}-\Delta\right), \\
& m_{h_{2}}^{2}=\frac{1}{2} v_{\chi}^{2}\left(\lambda_{1} t^{2}+\lambda_{2}+\Delta\right),
\end{aligned}
$$

where $t \equiv \frac{v_{\rho}}{v_{\chi}}$ and

$$
\Delta=\left(\lambda_{1} t^{2}-\lambda_{2}\right)^{2}-\lambda_{3}^{2} t^{2} .
$$

The corresponding eigenvectors are

$$
\left(\begin{array}{l}
h_{1} \\
h_{2}
\end{array}\right)=\left(\begin{array}{cc}
c_{\beta} & -s_{\beta} \\
s_{\beta} & c_{\beta}
\end{array}\right)\left(\begin{array}{l}
R_{\rho} \\
R_{\chi}
\end{array}\right)
$$

with

$$
c_{\beta}=\frac{1}{\sqrt{2}}\left(1+\frac{\lambda_{1} t^{2}-\lambda_{2}}{\sqrt{\Delta}}\right)^{\frac{1}{2}}, \quad s_{\beta}=\frac{1}{\sqrt{2}}\left(1-\frac{\lambda_{1} t^{2}-\lambda_{2}}{\sqrt{\Delta}}\right)^{\frac{1}{2}} .
$$

In the neutral pseudoscalar sector, there are two Goldstones bosons $I_{\rho}$ and $I_{\chi}$ which are eaten by the neutral gauge bosons $Z$ and $Z^{\prime}$, respectively.

In the effective limit: $v_{\chi} \gg v_{\rho}$ we have

$$
\begin{aligned}
& c_{\alpha} \approx 1, s_{\alpha} \approx 0, \quad \sqrt{\Delta} \approx \lambda_{2}-\lambda_{1} t^{2}+\frac{\lambda_{3}^{2}}{2 \lambda_{2}} t^{2}, \\
& c_{\beta} \approx \frac{1}{\sqrt{2}}\left(1+\frac{\lambda_{3} t^{2}}{\lambda_{1} t^{2}-\lambda_{2}}\right)^{\frac{1}{2}}, \quad s_{\beta} \approx \frac{1}{\sqrt{2}}\left(1-\frac{\lambda_{3} t^{2}}{\lambda_{1} t^{2}-\lambda_{2}}\right)^{\frac{1}{2}}
\end{aligned}
$$

This gives the following consequences:

(1) $\tilde{h}^{--} \approx \chi^{--}$and $h^{++} \approx \rho^{++}$.

(2) Masses of neutral Higgs bosons

$$
m_{h_{1}}^{2}=\left(\lambda_{1}-\frac{\lambda_{3}^{2}}{4 \lambda_{2}}\right) v_{\rho}^{2}, \quad m_{h_{2}}^{2}=\lambda_{2} v_{\chi}^{2}+\frac{\lambda_{3}^{2}}{4 \lambda_{2}} v_{\rho}^{2},
$$

(3) The positiveness of masses yields: $\lambda_{1}>0, \lambda_{2}>0,4 \lambda_{1} \lambda_{2}>\lambda_{3}^{2}$. 
Let us resume content of the Higgs sector: the physical scalar spectrum of the RM331 model is composed by a doubly charged scalar $h^{++}$and two neutral scalars $h_{1}$ and $h_{2}$. Since the lightest neutral field, $h_{1}$, is basically a $S U(2)_{L}$ component in the linear combination Eq. (17), we identify it as the standard Higgs boson in this model. Thus

$$
\rho=\left(\begin{array}{c}
G_{W^{+}} \\
\frac{v_{\rho}}{\sqrt{2}}+\frac{1}{\sqrt{2}}\left(h_{1}+i G_{Z}\right) \\
h^{++}
\end{array}\right), \quad \chi=\left(\begin{array}{c}
G_{V^{-}} \\
G_{U^{--}} \\
\frac{v_{\chi}}{\sqrt{2}}+\frac{1}{\sqrt{2}}\left(h_{2}+i G_{Z^{\prime}}\right)
\end{array}\right)
$$

Note that $h^{--}$carries lepton number two. Therefore it is scalar bilepton.

\section{GAUGE BOSONS}

In order to obtain the expression for the masses of the massive gauge bosons of the model, we have to substitute the expansion of the Eq. (8) in the Lagrangian,

$$
\mathcal{L}=\left(\mathcal{D}_{\mu} \chi\right)^{\dagger}\left(\mathcal{D}^{\mu} \chi\right)+\left(\mathcal{D}_{\mu} \rho\right)^{\dagger}\left(\mathcal{D}^{\mu} \rho\right),
$$

where,

$$
D_{\mu}=\partial_{\mu}-i g A_{\mu}^{a} \frac{\lambda^{a}}{2}-i g_{X} X \frac{\lambda_{9}}{2} B_{\mu}
$$

with $\lambda_{9}=\sqrt{\frac{2}{3}} \operatorname{diag}(1,1,1)$ so that $\operatorname{Tr}\left(\lambda_{9} \lambda_{9}\right)=2$. The coupling constants of $S U(3)_{L}$ and $U(1)_{X}$ satisfy the following relation

$$
\frac{g_{X}^{2}}{g^{2}}=\frac{6 s_{W}^{2}}{1-4 s_{W}^{2}}
$$

where the following notations are used $c_{W}=\cos \theta_{W}, s_{W}=\sin \theta_{W}, t_{W}=\tan \theta_{W}$ with $\theta_{W}$ being the Weinberg mixing angle. The eigenstates of the charged gauge bosons and their respective masses are given by,

$$
\begin{aligned}
& W^{ \pm}=\frac{A^{1} \mp i A^{2}}{\sqrt{2}} \rightarrow M_{W^{ \pm}}^{2}=\frac{g^{2} v_{\rho}^{2}}{4}, \\
& V^{ \pm}=\frac{A^{4} \pm i A^{5}}{\sqrt{2}} \rightarrow M_{V^{ \pm}}^{2}=\frac{g^{2} v_{\chi}^{2}}{4}, \\
& U^{ \pm \pm}=\frac{A^{6} \pm i A^{7}}{\sqrt{2}} \rightarrow M_{U^{ \pm \pm}}^{2}=\frac{g^{2}\left(v_{\rho}^{2}+v_{\chi}^{2}\right)}{4}
\end{aligned}
$$

From (25), it follows that $v_{\rho}=246 \mathrm{GeV}$. Note that there is mass splitting of the charged gauge bosons

$$
M_{U}^{2}-M_{V}^{2}=M_{W}^{2}
$$

The covariant derivative of the lepton triplets is

$$
\frac{g}{2} \vec{\lambda} \vec{A}_{\mu}=\left(\begin{array}{lcr}
\frac{g}{2}\left(A_{\mu}^{3}+\frac{1}{\sqrt{3}} A_{\mu}^{8}\right) & \frac{g}{\sqrt{2}} W_{\mu}^{+} & \frac{g}{\sqrt{2}} V_{\mu}^{-} \\
\frac{g}{\sqrt{2}} W_{\mu}^{-} & \frac{g}{2}\left(-A_{\mu}^{3}+\frac{1}{\sqrt{3}} A_{\mu}^{8}\right) & \frac{g}{\sqrt{2}} U_{\mu}^{--} \\
\frac{g}{\sqrt{2}} V_{\mu}^{+} & \frac{g}{\sqrt{2}} U_{\mu}^{++} & -g \frac{1}{\sqrt{3}} A_{\mu}^{8}
\end{array}\right) .
$$


while for the anti-triplets we have,

$$
\frac{g}{2} \vec{\lambda} \vec{A}_{\mu}=\left(\begin{array}{ccr}
-\frac{g}{2}\left(A_{\mu}^{3}+\frac{1}{\sqrt{3}} A_{\mu}^{8}\right) & -\frac{g}{\sqrt{2}} W_{\mu}^{-} & -\frac{g}{\sqrt{2}} V_{\mu}^{+} \\
-\frac{g}{\sqrt{2}} W_{\mu}^{+} & -\frac{g}{2}\left(-A_{\mu}^{3}+\frac{1}{\sqrt{3}} A_{\mu}^{8}\right) & -\frac{g}{\sqrt{2}} U_{\mu}^{++} \\
-\frac{g}{\sqrt{2}} V_{\mu}^{-} & -\frac{g}{\sqrt{2}} U_{\mu}^{--} & g \frac{1}{\sqrt{3}} A_{\mu}^{8}
\end{array}\right),
$$

where $\bar{\lambda}=-\lambda^{*}$.

In the neutral gauge boson sector, in the basis $\left(A_{\mu}^{3}, A_{\mu}^{8}, B_{\mu}\right)$, mass mixing matrix is given

$$
M^{2}=\frac{g^{2}}{4}\left(\begin{array}{ccc}
v_{\rho}^{2} & -\frac{v_{\rho}^{2}}{\sqrt{3}} & -2 t v_{\rho}^{2} \\
-\frac{v_{\rho}^{2}}{\sqrt{3}} & \frac{1}{3}\left(v_{\rho}^{2}+4 v_{\chi}^{2}\right) & \frac{2}{\sqrt{3}}\left(v_{\rho}^{2}+2 v_{\chi}^{2}\right) \\
-2 t v_{\rho}^{2} & \frac{2}{\sqrt{3}}\left(v_{\rho}^{2}+2 v_{\chi}^{2}\right) & 4 t^{2}\left(v_{\rho}^{2}+v_{\chi}^{2}\right)
\end{array}\right)
$$

where $t=\frac{g_{X}}{g}$. We can easily identify the photon field $A_{\mu}$ as well as the massive bosons $Z$ and $Z^{\prime}[9]$

$$
\begin{aligned}
& A_{\mu}=s_{W} A_{\mu}^{3}+c_{W}\left(\sqrt{3} t_{W} A_{\mu}^{8}+\sqrt{1-3 t_{W}^{2}} B_{\mu}\right), \\
& Z_{\mu}=c_{W} A_{\mu}^{3}-s_{W}\left(\sqrt{3} t_{W} A_{\mu}^{8}+\sqrt{1-3 t_{W}^{2}} B_{\mu}\right),
\end{aligned}
$$

and

$$
Z_{\mu}^{\prime}=-\sqrt{1-3 t_{W}^{2}} A_{\mu}^{8}+\sqrt{3} t_{W} B_{\mu}^{8}
$$

Masses of massive neutral gauge bosons are given by

$$
m_{Z}^{2}=\frac{g^{2}}{4 c_{W}^{2}} v_{\rho}^{2}, \quad m_{Z^{\prime}}^{2}=\frac{g^{2} c_{W}^{2}}{3\left(1-4 s_{W}^{2}\right)} v_{\chi}^{2} .
$$

For practical calculations, it is useful the following relations

$$
\begin{aligned}
& A_{\mu}^{3}=c_{W} Z_{\mu}+s_{W} A_{\mu} \\
& A_{\mu}^{8}=\sqrt{3} t_{W} s_{W} Z_{\mu}+\sqrt{1-3 t_{W}^{2}} Z_{\mu}^{\prime}-\sqrt{3} s_{W} A_{\mu}, \\
& B_{\mu}=-s_{W} \sqrt{1-3 t_{W}^{2}} Z_{\mu}+\sqrt{3} t_{W} Z_{\mu}^{\prime}+c_{W} \sqrt{1-3 t_{W}^{2}} A_{\mu} .
\end{aligned}
$$

Trilinear and quartic interactions of the gauge bosons are the same as in Ref. [10]

\section{FERMION MASSES}

With a shortened scalar content, the fermion masses originate from Yukawa couplings and another part are due to effective operators. The appropriate sources of mass for each fermion in the model are: The new quarks (exotic) get mass from the following Yukawa couplings,

$$
\begin{aligned}
L_{Y u k}^{\text {exot }}= & \lambda_{11}^{J} \bar{Q}_{1 L} \chi J_{1 R}+\lambda_{i j}^{J} \bar{Q}_{i L} \chi^{*} J_{j R}+H . c . \\
= & \lambda_{11}^{J}\left(\bar{u}_{1 L} \chi^{-}+\bar{d}_{1 L} \chi^{--}+\bar{J}_{1 L} \chi^{0}\right) J_{1 R} \\
& \lambda_{i j}^{J}\left(\bar{d}_{i L} \chi^{+}-\bar{u}_{i L} \chi^{++}+\bar{J}_{i L} \chi^{0 *}\right) J_{j R}+\text { H.c. }
\end{aligned}
$$


When the $\chi$ field develops its VEV, these couplings lead to the mass matrix in the basis $\left(J_{1}, J_{2}, J_{3}\right)$,

$$
M_{J}=\frac{v_{\chi}}{\sqrt{2}}\left(\begin{array}{ccc}
\lambda_{11}^{J} & 0 & 0 \\
0 & \lambda_{22}^{J} & \lambda_{23}^{J} \\
0 & \lambda_{32}^{J} & \lambda_{33}^{J}
\end{array}\right)
$$

which, after diagonalization, leads to mass eigenvalues at $v_{\chi} \sim$ few $\mathrm{TeV}$ scale.

As for the standard quarks, their masses come from a combination of renormalizable Yukawa interactions and specific effective dimension-five operators given by,

$$
\begin{aligned}
L_{Y u k}^{u} & =\lambda_{i a}^{u} \bar{Q}_{i L} \rho^{*} u_{a R}+\frac{\lambda_{1 a}^{u}}{\Lambda} \varepsilon_{n m p}\left(\bar{Q}_{1 L n} \rho_{m}^{*} \chi_{p}^{*}\right) u_{a R}+H . c . \\
& =\lambda_{i a}^{u}\left(\bar{d}_{i L} \rho^{-}-\bar{u}_{u L} \rho^{0 *}+\bar{J}_{i L} \rho^{--}\right) u_{a R} \\
& +\frac{\lambda_{1 a}^{u}}{\Lambda}\left[\bar{u}_{1 L}\left(\rho^{0 *} \chi^{0 *}-\rho^{--} \chi^{++}\right)+\bar{d}_{1 L}\left(\rho^{--} \chi^{+}-\rho^{-} \chi^{0 *}\right)\right. \\
& \left.+\bar{J}_{1 L}\left(\rho^{-} \chi^{++}-\rho^{0 *} \chi^{+}\right)\right] u_{a R}+H . c .
\end{aligned}
$$

In the basis $\left(u_{1}, u_{2}, u_{3}\right)$, the up-type quarks mass matrix takes the following form

$$
m_{u}=\frac{v_{\chi}}{\Lambda \sqrt{2}}\left(\begin{array}{ccc}
\lambda_{11}^{u} v_{\rho} & \lambda_{12}^{u} v_{\rho} & \lambda_{13}^{u} v_{\rho} \\
-\lambda_{21}^{u} v_{\rho} & -\lambda_{22}^{u} v_{\rho} & -\lambda_{23}^{u} v_{\rho} \\
-\lambda_{31}^{u} v_{\rho} & -\lambda_{32}^{u} v_{\rho} & -\lambda_{33}^{u} v_{\rho}
\end{array}\right)
$$

For down quark sector, the relevant Yukawa interactions are

$$
\begin{aligned}
L_{Y u k}^{d} & =\lambda_{1 a}^{d} \bar{Q}_{1 L} \rho d_{a R}+\frac{\lambda_{i a}^{d}}{\Lambda} \varepsilon_{n m p}\left(\bar{Q}_{i L n} \rho_{m} \chi_{p}\right) d_{a R}+H . c . \\
& =\lambda_{1 a}^{d}\left(\bar{u}_{1 L} \rho^{+}+\bar{d}_{1 L} \rho^{0}+\bar{J}_{1 L} \rho^{++}\right) d_{a R} \\
& +\frac{\lambda_{i a}^{d}}{\Lambda}\left[\bar{d}_{i L}\left(\rho^{0} \chi^{0}-\rho^{++} \chi^{--}\right)+\bar{u}_{i L}\left(\rho^{+} \chi^{0}-\rho^{++} \chi^{-}\right)\right. \\
& \left.+\bar{J}_{i L}\left(\rho^{+} \chi^{--}-\rho^{0} \chi^{-}\right)\right] d_{a R}+H . c .
\end{aligned}
$$

In the basis $\left(d_{1}, d_{2}, d_{3}\right)$, the mass matrix for the down-type quarks is,

$$
m_{d}=\frac{v_{\chi}}{\Lambda \sqrt{2}}\left(\begin{array}{ccc}
\lambda_{11}^{d} v_{\rho} & \lambda_{12}^{d} v_{\rho} & \lambda_{13}^{d} v_{\rho} \\
\lambda_{21}^{d} v_{\rho} & \lambda_{22}^{d} v_{\rho} & \lambda_{23}^{d} v_{\rho} \\
\lambda_{31}^{d} v_{\rho} & \lambda_{32}^{d} v_{\rho} & \lambda_{33}^{d} v_{\rho}
\end{array}\right)
$$

We remember that the highest energy scale where the model is found to be perturbatively reliable is about $\Lambda=4-5 \mathrm{TeV}$ [11]. There are 18 free Yukawa couplings to generate masses for 6 quarks only. Thus we have enough free parameters. For a naive analysis, it is just necessary to consider the diagonal case [6] where

$$
\begin{aligned}
& m_{u} \approx \lambda_{11}^{u} \frac{v_{\chi} v_{\rho}}{2 \Lambda}, \quad m_{d} \approx \lambda_{11}^{d} \frac{v_{\rho}}{\sqrt{2}}, \quad m_{s} \approx \lambda_{22}^{d} \frac{v_{\chi} v_{\rho}}{2 \Lambda}, \\
& m_{c} \approx-\lambda_{22}^{u} \frac{v_{\rho}}{\sqrt{2}}, \quad m_{b} \approx \lambda_{33}^{d} \frac{v_{\chi} v_{\rho}}{2 \Lambda}, \quad m_{t} \approx-\lambda_{33}^{u} \frac{v_{\rho}}{\sqrt{2}},
\end{aligned}
$$

where $m_{u}, m_{d}, \cdots$ represent the masses of the quarks up, down, .., respectively. For sake of simplicity let us assume that $\Lambda \approx v_{\chi}, \Lambda=5 \mathrm{TeV}, v_{\chi}=1 \mathrm{TeV}, m_{u}=2.5 \mathrm{MeV}, m_{d}=4.95$ 
$\mathrm{MeV}, m_{s}=105 \mathrm{MeV}, m_{c}=1.26 \mathrm{GeV}, m_{b}=4.25 \mathrm{GeV}$ and $m_{t}=179 \mathrm{GeV}$, we need [6] $\lambda_{11}^{u} \approx 10^{-3}, \lambda_{11}^{d} \approx 2.8 \times 10^{-5}, \lambda_{22}^{d} \approx 2.1 \times 10^{-2}, \lambda_{22}^{u} \approx-7.24 \times 10^{-3}, \lambda_{33}^{d} \approx 8.5 \times 10^{-1}$, $\lambda_{33}^{u} \approx-1.03$.

Note that to guarantee the proton stability, as in Ref. [12] we should impose a discrete $Z_{2}$ symmetry over the quark fields,

$$
Q_{a L} \rightarrow-Q_{a L}, \quad q_{a R} \rightarrow-q_{a R}
$$

Finally, the charged lepton masses arise from the effective dimension five operator $[6]$,

$$
\begin{aligned}
L_{Y u k}^{l} & =\frac{\kappa_{l}}{\Lambda}\left(\overline{f_{L}^{c}} \rho^{*}\right)\left(\chi^{\dagger} f_{L}\right)+H . c . \\
& =\frac{\kappa^{l}}{\Lambda}\left[\overline{\nu_{L}^{c}} \rho^{-}+\overline{l_{L}^{c}} \rho^{0 *}+\overline{l_{L}} \rho^{--}\right]\left[\nu_{L} \chi^{+}+l_{L} \chi^{++}+l_{L}^{c} \chi^{0 *}\right]+H . c .
\end{aligned}
$$

This non-renormalizable operator generates a mass term for the charged leptons $m_{l}=$ $\frac{v_{\chi}}{2 \Lambda} \kappa_{l} v_{\rho} \approx \frac{1}{2} \kappa_{l} v_{\rho}$. Thus, for $m_{e}=0.5 \mathrm{MeV}, m_{\mu}=105 \mathrm{MeV}, m_{\tau}=1.77 \mathrm{GeV}$, we get $k_{e}=2 \times 10^{-5}, k_{\mu}=4.3 \times 10^{-3}$ and $k_{\tau}=7.2 \times 10^{-2}$.

Generation for correct neutrino mass, in this model, is still open question.

\section{CONCLUSIONS}

In this paper, we have presented in details the 3-3-1 model with most economical particle content. The minimal Higgs sector (with just two triplets) is enough to provide masses for gauge bosons as well as all fermions, except the neutrinos. In contradiction with the previous minimal 3-3-1 model, all Higgs masses and eigenstates are exactly obtained. In many aspects, the model in similar to the minimal 3-3-1 model, but it contains an advantage: a huge number of free parameters is reduced. Therefore the predictiveness of the model under consideration is much improved.

Phenomenology of the model for the LHC will be presented elsewhere.

\section{ACKNOWLEDGMENTS}

This research is funded by Vietnam National Foundation for Science and Technology Development (NAFOSTED) under grant number 103.01-2011.63.

\section{REFERENCES}

[1] K. Nakamura et al. [Particle Data Group], J. Phys. G37 (2010) 075021.

[2] F. Pisano and V. Pleitez, Phys. Rev. D46 (1992) 410; P. H. Frampton, Phys. Rev. Lett. 69 (1992) 2889; R. Foot, O. F. Hernandez, F. Pisano and V. Pleitez, Phys. Rev. D47 (1993) 4158.

[3] M. Singer, J. W. F. Valle and J. Schechter, Phys. Rev. D22 (1980) 738; R. Foot, H. N. Long and Tuan A. Tran, Phys. Rev. D50 (1994) 34(R); J. C. Montero, F. Pisano and V. Pleitez, Phys. Rev. D47 (1993) 2918; H. N. Long, Phys. Rev. D54 (1996) 4691; Phys. Rev. D53, 437 (1996).

[4] W. A. Ponce, Y. Giraldo and L. A. Sanchez, Phys. Rev. D67 (2003) 075001

[5] P. V. Dong, H. N. Long, D. T. Nhung and D. V. Soa, Phys. Rev. D73 (2006) 035004; P. V. Dong, D. T. Huong, Tr. T. Huong and H. N. Long, Phys. Rev. D74 (2006) 053003; for a review, see: P. V. Dong and H. N. Long, Adv. High Energy Phys. 2008 (2008) 739492.

[6] J. G. Ferreira, Jr, P. R. D. Pinheiro, C. A. de S. Pires and P. S. Rodrigues da Silva, Phys. Rev. D84 (2011) 095019 
[7] M. B. Tully and G. C. Joshi, Phys. Rev. D64 (2001) 0113001

[8] D. Chang and H. N. Long, Phys. Rev. D73 (2006) 053006

[9] D. Ng, Phys. Rev. D49 (1994) 4805.

[10] H. N. Long, D. V. Soa, Nucl. Phys. B601 (2001) 361; D. T. Binh, D .T. Huong, T. T. Huong, H. N. Long, D. V. Soa, J. Phys. G29 (2003) 1213

[11] A. G. Dias, R. Martinez, V. Pleitez, Eur. Phys. J. C39 (2005), 101. See also, A. G. Dias, and V. Pleitez, Phys. Rev. D80 (2009) 056007.

[12] F. Queiroz, C. A. de S. Pires, and P. S. Rodrigues da Silva, Phys. Rev. D82 (2010) 065018.

Received 25 May 2012. 